\title{
Oscillation of Second-Order Nonlinear Delay Dynamic Equations with Damping on Time Scales
}

\author{
H. A. Agwa, Ahmed M. M. Khodier, and Heba A. Hassan \\ Department of Mathematics, Faculty of Education, Ain Shams University, Roxy, Cairo 11757, Egypt \\ Correspondence should be addressed to Heba A. Hassan; heba_ali70@yahoo.com \\ Received 6 April 2014; Accepted 4 June 2014; Published 22 June 2014 \\ Academic Editor: Pei-Guang Wang
}

Copyright ( 2014 H. A. Agwa et al. This is an open access article distributed under the Creative Commons Attribution License, which permits unrestricted use, distribution, and reproduction in any medium, provided the original work is properly cited.

\begin{abstract}
We use the generalized Riccati transformation and the inequality technique to establish some new oscillation criteria for the secondorder nonlinear delay dynamic equation with damping on a time scale $\mathbb{T}\left(r(t) g\left(x(t), x^{\Delta}(t)\right)\right)^{\Delta}+p(t) g\left(x(t), x^{\Delta}(t)\right)+q(t) f(x(\tau(t)))=$ 0 , where $r(t), p(t)$, and $q(t)$ are positive right dense continuous (rd-continuous) functions on $\mathbb{T}$. Our results improve and extend some results established by Zhang et al., 2011. Also, our results unify the oscillation of the second-order nonlinear delay differential equation with damping and the second-order nonlinear delay difference equation with damping. Finally, we give some examples to illustrate our main results.
\end{abstract}

\section{Introduction}

The purpose of this paper is to give several oscillation criteria for the second-order nonlinear delay dynamic equation with damping on a time scale $\mathbb{T}$

$$
\begin{aligned}
& \left(r(t) g\left(x(t), x^{\Delta}(t)\right)\right)^{\Delta}+p(t) g\left(x(t), x^{\Delta}(t)\right) \\
& \quad+q(t) f(x(\tau(t)))=0,
\end{aligned}
$$

subject to the following hypotheses.

$\left(H_{1}\right) \mathbb{T}$ is a time scale which is unbounded above and $t_{0} \in \mathbb{T}$ with $t_{0}>0$. The time scale interval $\left[t_{0}, \infty\right)_{\mathbb{T}}$ is defined by $\left[t_{0}, \infty\right)_{\mathbb{T}}=\left[t_{0}, \infty\right) \cap \mathbb{T}$.

$\left(H_{2}\right) r(t), p(t)$, and $q(t)$ are positive right dense continuous functions on $\mathbb{T}$ such that $-p / r \in \mathscr{R}^{+}$and

$$
\int_{t_{0}}^{\infty}\left[\frac{1}{r(t)} e_{-p / r}\left(t, t_{0}\right)\right] \Delta t=\infty
$$

or

$$
\int_{t_{0}}^{\infty}\left[\frac{1}{r(t)} e_{-p / r}\left(t, t_{0}\right)\right] \Delta t<\infty
$$

$\left(H_{3}\right)$ Consider $f \in C(\mathbb{R}, \mathbb{R}), x f(x)>0$ for all $x \neq 0$ and there exists a positive constant $L$ such that $f(x) / x \geq$ $L$.
$\left(H_{4}\right)$ Consider $g \in C^{1}\left(\mathbb{R}^{2}, \mathbb{R}\right), v g(u, v)>0$ for all $v \neq 0$ and for any fixed $u \in \mathbb{R}$ and there exist positive constants $K_{1}, K_{2}$ such that

$$
K_{1} \leq \frac{g(u, v)}{v} \leq K_{2}
$$

$\left(H_{5}\right) \tau: \mathbb{T} \rightarrow \mathbb{T}$ is a strictly increasing and differentiable function such that

$$
\tau(t) \leq t, \quad \lim _{t \rightarrow \infty} \tau(t)=\infty .
$$

By a solution of (1), we mean that a nontrivial real valued function $x$ satisfies (1) for $t \in \mathbb{T}$. A solution $x$ of (1) is called oscillatory if it is neither eventually positive nor eventually negative. Otherwise, it is called nonoscillatory. Equation (1) is said to be oscillatory if all of its solutions are oscillatory. In this work, we study the solutions of (1) which are not identically vanishing eventually.

Many results have been obtained on the oscillation and nonoscillation of dynamic equations on time scales (see e.g., the papers [1-17], the books $[18,19]$ and the references cited 
therein). It is easy to see that (1) can be transformed into the equation

$$
\begin{array}{r}
\left(r(t) x^{\Delta}(t)\right)^{\Delta}+p(t) x^{\Delta}(t)+q(t) f(x(\tau(t)))=0, \\
t \in \mathbb{T}, \quad t \geq t_{0},
\end{array}
$$

where $g\left(x, x^{\Delta}\right)=x^{\Delta}$. If $r(t)=1, f(x)=x$, and $\tau(t)=t$, then (6) is simplified to the equation

$$
x^{\Delta \Delta}(t)+p(t) x^{\Delta}(t)+q(t) x(t)=0, \quad t \in \mathbb{T}, t \geq t_{0} .
$$

Also, if $p(t)=0,(6)$ is simplified to the equation

$$
\left(r(t) x^{\Delta}(t)\right)^{\Delta}+q(t) f(x(\tau(t)))=0, \quad t \in \mathbb{T}, t \geq t_{0} .
$$

If $r(t)=1,(8)$ takes the form

$$
x^{\Delta \Delta}(t)+q(t) f(x(\tau(t)))=0, \quad t \in \mathbb{T}, t \geq t_{0} .
$$

If $f(x)=x,(9)$ becomes

$$
x^{\Delta \Delta}(t)+q(t) x(\tau(t))=0, \quad t \in \mathbb{T}, t \geq t_{0} .
$$

In 2002, Guseinov and Kaymakçalan [10] studied (7) and established some sufficient conditions for nonoscillation. They proved that if

$$
\int_{t_{0}}^{\infty} p(t) \Delta t<\infty, \quad \int_{t_{0}}^{\infty} \operatorname{tq}(t) \Delta t<\infty
$$

then (7) is nonoscillatory. In 2005, Agarwal et al. [2] studied the linear delay dynamic equation (10) and Şahiner [12] considered the nonlinear delay dynamic equation (9) and gave some sufficient conditions for oscillation of (10) and (9). In 2007, Erbe et al. [8] considered the general nonlinear delay dynamic equations (8). They obtained some oscillation criteria which improve the results given by Şahiner [12]. In 2011, Zhang and Gao [16] considered the oscillation of solutions of second-order nonlinear delay dynamic equation (6) with damping and establishing some new results. In this paper, we use the generalized Riccati transformation and the inequality technique to obtain some new oscillation criteria for (1). Our results generalize and improve the results in [16].

This paper is organized as follows. In Section 2, we present some preliminaries on time scales. In Section 3, we give some lemmas that we need through our work. In Section 4, we establish some new sufficient conditions for oscillation of (1). Finally, in Section 5, we present some examples to illustrate our results.

\section{Some Preliminaries on Time Scales}

A time scale $\mathbb{T}$ is an arbitrary nonempty closed subset of the real numbers $\mathbb{R}$. On any time scale $\mathbb{T}$, we define the forward and backward jump operators by

$$
\sigma(t)=\inf \{s \in \mathbb{T}, s>t\}, \quad \rho(t)=\sup \{s \in \mathbb{T}, s<t\} .
$$

A point $t \in \mathbb{T}, t>\inf \mathbb{\mathbb { T }}$ is said to be left dense if $\rho(t)=t$, right dense if $t<\sup \mathbb{T}$ and $\sigma(t)=t$, left-scattered if $\rho(t)<t$, and right-scattered if $\sigma(t)>t$. The graininess function $\mu$ for a time scale $\mathbb{T}$ is defined by $\mu(t)=\sigma(t)-t$. The set $\mathbb{T}^{k}$ is derived from the time scale $\mathbb{T}$ as $\mathbb{T}^{k}=\mathbb{T}-m$ if $\mathbb{T}$ has a left-scattered maximum $m$. Otherwise, $\mathbb{T}^{k}=\mathbb{T}$.

A function $f: \mathbb{T} \rightarrow \mathbb{R}$ is called rd-continuous provided that it is continuous at right dense points of $\mathbb{T}$ and its left-sided limits exist at left dense points of $\mathbb{T}$. The set of rd-continuous functions is denoted by $C_{\text {rd }}(\mathbb{T}, \mathbb{R})$. By $C_{\text {rd }}^{1}(\mathbb{T}, \mathbb{R})$, we mean the set of functions whose delta derivative belong to $C_{\mathrm{rd}}(\mathbb{T}, \mathbb{R})$.

A function $f: \mathbb{T} \rightarrow \mathbb{R}$ is regressive provided that

$$
1+\mu(t) f(t) \neq 0, \quad \forall t \in \mathbb{T}^{k},
$$

holds. The set of all regressive and rd-continuous functions $f: \mathbb{T} \rightarrow \mathbb{R}$ is denoted by

$$
\mathscr{R}=\mathscr{R}(\mathbb{T})=\mathscr{R}(\mathbb{T}, \mathbb{R}) .
$$

If $q \in \mathscr{R}$, then we define the exponential function $e_{q}(t, s)$ by

$$
e_{q}(t, s)=\exp \left(\int_{s}^{t} \xi_{\mu(\tau)}(q(\tau)) \Delta \tau\right), \quad \text { for } s, t \in \mathbb{T},
$$

where the cylinder function $\xi_{h}(z)$ is defined by

$$
\xi_{h}(z)=\frac{1}{h} \log (1+z h) .
$$

For a function $f: \mathbb{T} \rightarrow \mathbb{R}$ (the range $\mathbb{R}$ of $f$ may be actually replaced by any Banach space), the delta derivative $f^{\Delta}$ is defined by

$$
f^{\Delta}(t)=\frac{f(\sigma(t))-f(t)}{\sigma(t)-t}
$$

provided $f$ is continuous at $t$ and $t$ is right-scattered. If $t$ is not right-scattered, then the delta derivative $f^{\Delta}(t)$ is defined by

$$
f^{\Delta}(t)=\lim _{s \rightarrow t^{+}} \frac{f(\sigma(t))-f(t)}{t-s}=\lim _{s \rightarrow t^{+}} \frac{f(t)-f(s)}{t-s}
$$

provided this limit exists.

A function $f:[a, b] \rightarrow \mathbb{R}$ is said to be differentiable if its derivative exists. The derivative $f^{\Delta}$ and the shift $f^{\sigma}$ of a function $f$ are related by the equation

$$
f^{\sigma}=f(\sigma(t))=f(t)+\mu(t) f^{\Delta}(t) .
$$

The delta derivative rules of the product $f g$ and the quotient $f / g$ (where $g g^{\sigma} \neq 0$ ) of two differentiable functions $f$ and $g$ are given by

$$
\begin{aligned}
(f \cdot g)^{\Delta}(t) & =f^{\Delta}(t) g(t)+f^{\sigma}(t) g^{\Delta}(t) \\
& =f(t) g^{\Delta}(t)+f^{\Delta}(t) g^{\sigma}(t), \\
\left(\frac{f}{g}\right)^{\Delta}(t) & =\frac{f^{\Delta}(t) g(t)-f(t) g^{\Delta}(t)}{g(t) g^{\sigma}(t)} .
\end{aligned}
$$


An integration by parts formula reads

$$
\int_{a}^{b} f(t) g^{\Delta}(t) \Delta t=[f(t) g(t)]_{a}^{b}-\int_{a}^{b} f^{\Delta}(t) g^{\sigma}(t) \Delta t
$$

or

$$
\int_{a}^{b} f^{\sigma}(t) g^{\Delta}(t) \Delta t=[f(t) g(t)]_{a}^{b}-\int_{a}^{b} f^{\Delta}(t) g(t) \Delta t
$$

and the infinite integral is defined by

$$
\int_{b}^{\infty} f(s) \Delta s=\lim _{t \rightarrow \infty} \int_{b}^{t} f(s) \Delta s
$$

Throughout this paper, we use

$$
d_{-}(t)=: \max \{0,-d(t)\}, \quad \alpha(t):=\frac{R(t)}{R(t)+\mu(t)},
$$

where $R(t)=k r(t) \int_{t_{0}}^{t}(\Delta s / r(s))$, and positive constants

$$
k=\frac{K_{1}}{K_{2}}, \quad \eta=\frac{K_{2}}{L},
$$

where $L, K_{1}$, and $K_{2}$ are defined in $\left(H_{3}\right),\left(H_{4}\right)$.

\section{Several Lemmas}

In this section, we present some lemmas that we need to prove our results in the next section.

Lemma 1. (Bohner and Peterson [18, Chapter 2]). If $g: \mathbb{T} \rightarrow$ $\mathbb{R}$ is $r d$-continuous such that $1+\mu(t) g(t)>0$ for all $t \in$ $\left[t_{0}, \infty\right)_{\mathbb{T}}$, then the initial value problem $y^{\Delta}=g(t) y, y\left(t_{0}\right)=$ $y_{0} \in \mathbb{R}$ has a unique and positive solution on $\left[t_{0}, \infty\right)_{\mathbb{T}}$, denoted bye $e_{g}\left(\cdot, t_{0}\right)$.

Lemma 2. (Bohner [5, Lemma 2]). For nonnegative $p$ with $-p \in \mathscr{R}^{+}$, one has the inequality

$$
1-\int_{s}^{t} p(u) \Delta u \leq e_{-p}(t, s), \quad \forall t \geq s .
$$

Lemma 3. If $\left(H_{1}\right)-\left(H_{5}\right)$, (2) hold and (1) has a positive solution $x$ on $\left[t_{0}, \infty\right)_{\mathbb{T}}$, then $\left(r(t) g\left(x, x^{\Delta}\right)\right)^{\Delta}<0, x^{\Delta}(t)>0$, and $x(t)>\alpha(t) x(\sigma(t))$ for $t \in\left[t_{0}, \infty\right)_{\mathbb{W}}$.

Proof. Since $x$ is a positive solution of $(1)$ on $\left[t_{0}, \infty\right)_{\mathbb{T}}$, we have

$$
\begin{aligned}
& \left(r(t) g\left(x(t), x^{\Delta}(t)\right)\right)^{\Delta}+p(t) g\left(x(t), x^{\Delta}(t)\right) \\
& \quad=-q(t) f(x(\tau(t)))<0 .
\end{aligned}
$$

Therefore,

$$
\begin{aligned}
& {\left[\frac{r(t) g\left(x(t), x^{\Delta}(t)\right)}{e_{-p / r}\left(\cdot, t_{0}\right)}\right]^{\Delta}} \\
& =\left(\left(r(t) g\left(x(t), x^{\Delta}(t)\right)\right)^{\Delta} e_{-p / r}\left(\cdot, t_{0}\right)\right. \\
& \left.\quad-e_{-p / r}^{\Delta}\left(\cdot, t_{0}\right) r(t) g\left(x(t), x^{\Delta}(t)\right)\right) \\
& \quad \times\left(e_{-p / r}\left(\cdot, t_{0}\right) e_{-p / r}^{\sigma}\left(\cdot, t_{0}\right)\right)^{-1} \\
& =\left(\left(r(t) g\left(x(t), x^{\Delta}(t)\right)\right)^{\Delta}+p(t) g\left(x(t), x^{\Delta}(t)\right)\right. \\
& \quad \times\left(e_{-p / r}^{\sigma}\left(\cdot, t_{0}\right)\right)^{-1}<0 .
\end{aligned}
$$

Hence,

$$
\frac{r(t) g\left(x(t), x^{\Delta}(t)\right)}{e_{-p / r}\left(\cdot, t_{0}\right)} \text { is decreasing. }
$$

We claim that $x^{\Delta}(t)>0$ on $\left[t_{0}, \infty\right)_{\mathbb{T}}$. If not, then there is $t \geq t_{1}$ such that

$$
\frac{r(t) g\left(x(t), x^{\Delta}(t)\right)}{e_{-p / r}\left(t, t_{0}\right)} \leq \frac{r\left(t_{1}\right) g\left(x\left(t_{1}\right), x^{\Delta}\left(t_{1}\right)\right)}{e_{-p / r}\left(t_{1}, t_{0}\right)}=: c<0 .
$$

By $\left(H_{4}\right)$, we get

$$
x^{\Delta}(t) \leq \frac{c}{K_{1}} \frac{1}{r(t)} e_{-p / r}\left(t, t_{0}\right), \quad K_{1}>0 .
$$

Integrating from $t_{1}$ to $t$, we get

$$
x(t) \leq x\left(t_{1}\right)+\frac{c}{K_{1}} \int_{t_{1}}^{t}\left[\frac{1}{r(s)} e_{-p / r}\left(s, t_{0}\right)\right] \Delta s .
$$

This implies that $x(t)$ is eventually negative which is a contradiction. Hence, $x^{\Delta}(t)>0$ on $\left[t_{0}, \infty\right)_{\mathbb{T}}$. Therefore,

$$
\begin{aligned}
x(t) & >x(t)-x\left(t_{1}\right) \\
& =\int_{t_{1}}^{t} x^{\Delta}(s) \Delta s \\
& >\int_{t_{1}}^{t} \frac{1}{K_{2}} g\left(x(s), x^{\Delta}(s)\right) \Delta s \quad \text { by }\left(H_{3}\right) \\
& >\frac{1}{K_{2}} \int_{t_{1}}^{t} \frac{r(s) g\left(x(s), x^{\Delta}(s)\right)}{r(s)} \Delta s, \quad K_{2}>0 .
\end{aligned}
$$


Using the fact that $r(t) g\left(x(t), x^{\Delta}(t)\right)$ is strictly decreasing, we get

$$
\begin{aligned}
x(t) & >\frac{r(t) g\left(x(t), x^{\Delta}(t)\right)}{K_{2}} \int_{t_{1}}^{t} \frac{\Delta s}{r(s)} \\
& >\frac{K_{1}}{K_{2}} r(t) x^{\Delta}(t) \int_{t_{1}}^{t} \frac{\Delta s}{r(s)}, \quad K_{1}>0 \quad\left(\text { by }\left(H_{3}\right)\right) \\
& >k r(t) x^{\Delta}(t) \int_{t_{1}}^{t} \frac{\Delta s}{r(s)}=R(t) x^{\Delta}(t), \quad \text { for } k=\frac{K_{1}}{K_{2}}>0,
\end{aligned}
$$

where $R(t)=k r(t) \int_{t_{1}}^{t}(\Delta s / r(s))$.

Hence,

$$
\begin{array}{r}
\frac{x(t)}{x^{\sigma}(t)}=\frac{x(t)}{x(t)+\mu(t) x^{\Delta}(t)} \geq \frac{R(t)}{R(t)+\mu(t)}=\alpha(t) \\
\text { on }\left[t_{0}, \infty\right)_{\mathbb{T}} .
\end{array}
$$

\section{Main Results}

Here, we establish some new sufficient conditions for oscillation of (1).

Theorem 4. Assume that $\left(H_{1}\right)-\left(H_{5}\right)$, (2) hold and $\tau \in$ $C_{r d}^{1}\left(\left[t_{0}, \infty\right)_{\mathbb{T}}, \mathbb{T}\right), \tau\left(\left[t_{0}, \infty\right)_{\mathbb{T}}\right)=\left[t_{0}, \infty\right)_{\mathbb{T}}$. If there exists a positive $\Delta$-differentiable function $\delta(t)$ such that

$$
\begin{aligned}
\limsup _{t \rightarrow \infty} \int_{t_{0}}^{t} & {[\alpha(\tau(s)) q(s)} \\
& \left.\quad-\frac{\eta r(\tau(s))}{4 \alpha(\tau(s)) \tau^{\Delta}(s)}\left(\frac{\delta^{\Delta}(s)}{\delta^{\sigma}(s)}-\frac{p(s)}{r(s)} \alpha(\tau(s))\right)^{2}\right] \\
& \times \delta^{\sigma}(s) \Delta s=\infty,
\end{aligned}
$$

then every solution of $(1)$ is oscillatory on $\left[t_{0}, \infty\right)_{\mathbb{T}}$.

Proof. Assume that (1) has a nonoscillatory solution $x(t)$ on $\left[t_{0}, \infty\right)_{\mathbb{T}}$. Also, assume that $x(t)>0, x(\tau(t))>0$ for all $t \in\left[t_{1}, \infty\right)_{\mathbb{T}}, t_{1} \in\left[t_{0}, \infty\right)_{\mathbb{W}}$. Consider the generalized Riccati substitution

$$
w(t)=\delta(t) \frac{r(t) g\left(x(t), x^{\Delta}(t)\right)}{x(\tau(t))}>0 .
$$

Using the delta derivative rules of product and quotient of two functions, we have

$$
\begin{aligned}
w^{\Delta}(t)= & \delta^{\Delta}(t) \frac{r(t) g\left(x(t), x^{\Delta}(t)\right)}{x(\tau(t))} \\
& +\delta^{\sigma}(t)\left(\frac{r(t) g\left(x(t), x^{\Delta}(t)\right)}{x(\tau(t))}\right)^{\Delta} \\
= & \frac{\delta^{\Delta}(t)}{\delta(t)} w(t)+\delta^{\sigma}(t)
\end{aligned}
$$

$$
\begin{aligned}
\times( & x(\tau(t))\left(r(t) g\left(x(t), x^{\Delta}(t)\right)\right)^{\Delta} \\
& \left.-(x(\tau(t)))^{\Delta}\left(r(t) g\left(x(t), x^{\Delta}(t)\right)\right)\right) \\
& \left.\times\left(x(\tau(t)) x^{\sigma}(\tau(t))\right)^{-1}\right) \\
=\frac{\delta^{\Delta}(t)}{\delta(t)} & w(t)+\delta^{\sigma}(t) \\
\times( & -\frac{p(t) g\left(x(t), x^{\Delta}(t)\right)}{x^{\sigma}(\tau(t))} \\
& -\frac{q(t) f(x(\tau(t)))}{x^{\sigma}(\tau(t))} \\
& \left.-\frac{x^{\Delta}(\tau(t)) \tau^{\Delta}(t)}{\delta(t) x^{\sigma}(\tau(t))} w(t)\right) .
\end{aligned}
$$

From the definition of $w(t)$, we have

$$
\begin{aligned}
& w^{\Delta}(t)=\frac{\delta^{\Delta}(t)}{\delta(t)} w(t) \\
&+\delta^{\sigma}(t)\left(-\frac{p(t) x(\tau(t))}{\delta(t) r(t) x^{\sigma}(\tau(t))} w(t)\right. \\
&-\frac{q(t) f(x(\tau(t)))}{x^{\sigma}(\tau(t))} \\
& \\
&\left.\quad-\frac{x^{\Delta}(\tau(t)) \tau^{\Delta}(t)}{\delta(t) x^{\sigma}(\tau(t))} w(t)\right) .
\end{aligned}
$$

Using the fact $f(x) / x \geq L$ and $x(t) / x^{\sigma}(t)>\alpha(t)$, we get

$$
\begin{aligned}
w^{\Delta}(t) \leq & \frac{\delta^{\Delta}(t)}{\delta(t)} w(t) \\
& \\
& +\delta^{\sigma}(t)\left(-\frac{p(t) \alpha(\tau(t))}{\delta(t) r(t)} w(t)-\operatorname{Lq}(t) \alpha(\tau(t))\right. \\
& \left.-\frac{x^{\Delta}(\tau(t)) \tau^{\Delta}(t)}{\delta(t) x^{\sigma}(\tau(t))} w(t)\right) .
\end{aligned}
$$

Integrating the inequality $\left(r(t) g\left(x, x^{\Delta}\right)\right)^{\Delta}<0$ from $\tau(t)$ to $t$, using the definition of $w(t)$ and $\left(H_{3}\right)$, we get

$$
x^{\Delta}(\tau(t))>\frac{x(\tau(t))}{K_{2} \delta(t) r(\tau(t))} w(t) .
$$


Now, substituting (41) in (40), we have

$$
\begin{aligned}
& w^{\Delta}(t) \leq \frac{\delta^{\Delta}(t)}{\delta(t)} w(t) \\
&+\delta^{\sigma}(t)\left(-\frac{p(t) \alpha(\tau(t))}{\delta(t) r(t)} w(t)\right. \\
&-L q(t) \alpha(\tau(t)) \\
&\left.\quad-\frac{\alpha(\tau(t)) \tau^{\Delta}(t)}{K_{2} \delta^{2}(t) r(\tau(t))} w^{2}(t)\right) .
\end{aligned}
$$

Hence,

$$
\begin{aligned}
w^{\Delta}(t) \leq & -L q(t) \delta^{\sigma}(t) \alpha(\tau(t)) \\
& +\left(\frac{\delta^{\Delta}(t)}{\delta(t)}-\frac{p(t) \delta^{\sigma}(t) \alpha(\tau(t))}{\delta(t) r(t)}\right) w(t) \\
& -\frac{\delta^{\sigma}(t) \alpha(\tau(t)) \tau^{\Delta}(t)}{K_{2} \delta^{2}(t) r(\tau(t))} w^{2}(t) \\
= & -L q(t) \delta^{\sigma}(t) \alpha(\tau(t)) \\
& +G(t) \frac{w(t)}{\delta(t)}-Q(t) \frac{w^{2}(t)}{\delta^{2}(t)},
\end{aligned}
$$

where $G(t)=\delta^{\Delta}(t)-p(t) \delta^{\sigma}(t) \alpha(\tau(t)) / r(t)$ and $Q(t)=$ $\delta^{\sigma}(t) \alpha(\tau(t)) \tau^{\Delta}(t) / K_{2} r(\tau(t))$.

Therefore,

$$
\begin{aligned}
w^{\Delta}(t) \leq & -L q(t) \delta^{\sigma}(t) \alpha(\tau(t)) \\
& -\left(\frac{\sqrt{Q(t)}}{\delta(t)} w(t)-\frac{G(t)}{2 \sqrt{Q(t)}}\right)^{2}+\frac{G^{2}(t)}{4 Q(t)} \\
\leq & -L q(t) \delta^{\sigma}(t) \alpha(\tau(t))+\frac{G^{2}(t)}{4 Q(t)} .
\end{aligned}
$$

Hence,

$$
\begin{aligned}
& w^{\Delta}(t) \\
& \leq-\operatorname{Lq}(t) \delta^{\sigma}(t) \alpha(\tau(t)) \\
& \quad+\frac{K_{2}\left(\delta^{\Delta}(t)-(p(t) / r(t)) \delta^{\sigma}(t) \alpha(\tau(t))\right)^{2} r(\tau(t))}{4 \delta^{\sigma}(t) \alpha(\tau(t)) \tau^{\Delta}(t)} .
\end{aligned}
$$

Integrating the above inequality from $t_{0}$ to $t$, we obtain

$$
\begin{aligned}
& \int_{t_{0}}^{t}\left[\alpha(\tau(s)) q(s)-\frac{\eta r(\tau(s))}{4 \alpha(\tau(s)) \tau^{\Delta}(s)}\right. \\
& \left.\quad \times\left(\frac{\delta^{\Delta}(s)}{\delta^{\sigma}(s)}-\frac{p(s)}{r(s)} \alpha(\tau(s))\right)^{2}\right] \delta^{\sigma}(s) \Delta s \\
& \leq \frac{1}{L}\left(w\left(t_{0}\right)-w(t)\right) \leq \frac{1}{L} w\left(t_{0}\right),
\end{aligned}
$$

and taking the limit supremum as $t \rightarrow \infty$, we obtain a contradiction to condition (36). Therefore, every solution of (1) is oscillatory on $\left[t_{0}, \infty\right)_{\mathbb{T}}$.

Theorem 5. Assume that $\left(H_{1}\right)-\left(H_{5}\right)$ and (2) hold. Let $H$ be an rd-continuous function defined as follows:

$$
H: D_{\mathbb{T}} \equiv\left\{(t, s): t \geq s \geq t_{0}, t, s \in\left[t_{0}, \infty\right)_{\mathbb{T}}\right\} \longrightarrow \mathbb{R},
$$

such that

$$
\begin{gathered}
H(t, t)=0, \quad \text { for } t \geq t_{0}, \\
H(t, s)>0, \quad \text { for } t>s \geq t_{0}, \\
t, s \in\left[t_{0}, \infty\right)_{\mathbb{T}}
\end{gathered}
$$

and $H$ has a nonpositive continuous $\Delta$-partial derivative $H^{\Delta_{s}}(t, s)$. If there exists a positive $\Delta$-differentiable function $\delta(t)$ such that

$$
\begin{aligned}
& \limsup _{t \rightarrow \infty} \frac{1}{H\left(t, t_{0}\right)} \\
& \times \int_{t_{0}}^{t} H(t, s)\left[\alpha(\tau(s)) q(s)-\frac{\eta r(\tau(s))}{4 \alpha(\tau(s)) \tau^{\Delta}(s)}\right. \\
&\left.\times\left(\frac{\delta^{\Delta}(s)}{\delta^{\sigma}(s)}-\frac{p(s)}{r(s)} \alpha(\tau(s))\right)^{2}\right] \\
& \times \delta^{\sigma}(s) \Delta s=\infty,
\end{aligned}
$$

then every solution of (1) is oscillatory on $\left[t_{0}, \infty\right)_{\mathbb{T}}$.

Proof. Assume that (1) has a nonoscillatory solution $x(t)$ on $\left[t_{0}, \infty\right)_{\mathbb{T}}$. Also, assume that $x(t)>0, x(\tau(t))>0$, for all $t \in\left[t_{1}, \infty\right)_{\mathbb{T}}, t_{1} \in\left[t_{0}, \infty\right)_{\mathbb{T}}$. We proceed as in the proof of Theorem 4 to get (45)

$$
\begin{aligned}
& \operatorname{Lq}(t) \delta^{\sigma}(t) \alpha(\tau(t)) \\
& \quad-\frac{K_{2}\left(\delta^{\Delta}(t)-(p(t) / r(t)) \delta^{\sigma}(t) \alpha(\tau(t))\right)^{2} r(\tau(t))}{4 \delta^{\sigma}(t) \alpha(\tau(t)) \tau^{\Delta}(t)} \\
& \quad \leq-w^{\Delta}(t) .
\end{aligned}
$$

Multiplying the above inequality by $H(t, s)$, integrating from $t_{0}$ to $t$ and using (48), we get

$$
\begin{aligned}
& \int_{t_{0}}^{t} H(t, s)[L \alpha(\tau(s)) q(s) \delta^{\sigma}(s) \\
&-\frac{K_{2} r(\tau(s))}{4 \delta^{\sigma}(s) \alpha(\tau(s)) \tau^{\Delta}(s)} \\
&\left.\times\left(\delta^{\Delta}(s)-\frac{p(s)}{r(s)} \delta^{\sigma}(s) \alpha(\tau(s))\right)^{2}\right] \Delta s \\
& \leq-\int_{t_{0}}^{t} H(t, s) w^{\Delta}(s) \Delta s
\end{aligned}
$$




$$
\begin{aligned}
& =-\left.H(t, s) w(s)\right|_{t_{0}} ^{t}+\int_{t_{0}}^{t} H^{\Delta_{s}}(t, s) w(\sigma(s)) \Delta s \\
& \leq H\left(t, t_{0}\right) w\left(t_{0}\right) .
\end{aligned}
$$

Thus,

$$
\begin{aligned}
\frac{1}{H\left(t, t_{0}\right)} \int_{t_{0}}^{t} H(t, s)[\alpha(\tau(s)) q(s) & \\
& -\frac{\eta r(\tau(s))}{4 \alpha(\tau(s)) \tau^{\Delta}(s)} \\
& \left.\times\left(\frac{\delta^{\Delta}(s)}{\delta^{\sigma}(s)}-\frac{p(s)}{r(s)} \alpha(\tau(s))\right)^{2}\right] \\
\times \delta^{\sigma}(s) \Delta s & \\
\leq \frac{1}{L} w\left(t_{0}\right)<\infty, &
\end{aligned}
$$

which is a contradiction to (49). This completes the proof.

Now, If $H(t, s)$ is a function defined by

$$
\begin{gathered}
H(t, s)=(t-s)^{m}, \\
m \geq 1, \quad t \geq s \geq t_{0}, \quad t, s \in\left[t_{0}, \infty\right)_{\mathbb{T}}
\end{gathered}
$$

then, we have the following result.

Corollary 6. Assume that $\left(H_{1}\right)-\left(H_{5}\right)$ and (2) hold. If there exists a positive $\Delta$-differentiable function $\delta(t)$ and $m \geq 1$ such that

$$
\begin{aligned}
& \limsup _{t \rightarrow \infty} \frac{1}{t^{m}} \int_{t_{0}}^{t}(t-s)^{m} \\
& \times\left[\alpha(\tau(s)) q(s)-\frac{\eta r(\tau(s))}{4 \alpha(\tau(s)) \tau^{\Delta}(s)}\right. \\
&\left.\quad \times\left(\frac{\delta^{\Delta}(s)}{\delta^{\sigma}(s)}-\frac{p(s)}{r(s)} \alpha(\tau(s))\right)^{2}\right] \\
& \quad \times \delta^{\sigma}(s) \Delta s=\infty,
\end{aligned}
$$

then every solution of $(1)$ is oscillatory on $\left[t_{0}, \infty\right)_{\mathbb{T}}$.

Theorem 7. Assume that $\left(H_{1}\right)-\left(H_{5}\right)$ and (2) hold. Let $H$ be an rd-continuous function defined as

$$
H: D_{\mathbb{T}} \equiv\left\{(t, s): t \geq s \geq t_{0}, t, s \in\left[t_{0}, \infty\right)_{\mathbb{T}}\right\} \longrightarrow \mathbb{R},
$$

such that

$$
\begin{gathered}
H(t, t)=0, \quad \text { for } t \geq t_{0}, \\
H(t, s)>0, \quad \text { for } t>s \geq t_{0}, \\
t, s \in\left[t_{0}, \infty\right)_{\mathbb{T}}
\end{gathered}
$$

and $H$ has a nompositive continuous $\Delta$-partial derivative $H^{\Delta_{s}}(t, s)$. Let $h: D_{\mathbb{T}} \rightarrow \mathbb{R}$ be an rd-continuous function satisfying

$$
-H^{\Delta_{s}}(t, s)=h(t, s) \sqrt{H(t, s)}, \quad \forall(t, s) \in D_{\mathbb{T}} .
$$

If there exists a positive nondecreasing $\Delta$-differentiable function $\delta(t)$ such that

$$
\begin{aligned}
& \limsup _{t \rightarrow \infty} \frac{1}{H\left(t, t_{0}\right)} \\
& \times \int_{t_{0}}^{t}\left[H(t, s) q(s) \alpha(\tau(s))-\frac{\eta r(\tau(s))}{4 \alpha(\tau(s)) \tau^{\Delta}(s)}\right. \\
& \quad \times\left(\frac{G(s)}{\delta^{\sigma}(s)} \sqrt{H(t, s)}\right. \\
&\left.\left.\quad+\frac{\delta(s)}{\delta^{\sigma}(s)} h_{-}(t, s)\right)^{2}\right] \\
& \quad \times \delta^{\sigma}(s) \Delta s=\infty
\end{aligned}
$$

where $G(t)=\delta^{\Delta}(t)-p(t) \delta^{\sigma}(t) \alpha(\tau(t)) / r(t)$, then every solution of (1) is oscillatory on $\left[t_{0}, \infty\right)_{\mathbb{T}}$.

Proof. Assume that (1) has a nonoscillatory solution $x(t)$ on $\left[t_{0}, \infty\right)_{\mathbb{T}}$. Also, assume that $x(t)>0, x(\tau(t))>0$, for all $t \in\left[t_{1}, \infty\right)_{\mathbb{T}}, t_{1} \in\left[t_{0}, \infty\right)_{\mathbb{T}}$ and there is $T \in\left[t_{0}, \infty\right)_{\mathbb{T}}$ such that $x(t)$ satisfies the conclusion of Lemma 3 on $[T, \infty)_{\mathbb{T}}$. Proceeding as in the proof of Theorem 4, we get (43) which has the form

$$
w^{\Delta}(t) \leq-L q(t) \delta^{\sigma}(t) \alpha(\tau(t))+G(t) \frac{w(t)}{\delta(t)}-Q(t) \frac{w^{2}(t)}{\delta^{2}(t)},
$$

where $G(t)=\delta^{\Delta}(t)-p(t) \delta^{\sigma}(t) \alpha(\tau(t)) / r(t)$ and $Q(t)=$ $\delta^{\sigma}(t) \alpha(\tau(t)) \tau^{\Delta}(t) / K_{2} r(\tau(t))$.

Multiplying the above inequality by $H(t, s)$, integrating from $t_{0}$ to $t$, and using (56) and (57), we get

$$
\begin{gathered}
\int_{t_{0}}^{t} L H(t, s) q(s) \delta^{\sigma}(s) \alpha(\tau(s)) \Delta s \\
\leq H\left(t, t_{0}\right) w\left(t_{0}\right) \\
\quad+\int_{t_{0}}^{t} H^{\Delta_{s}}(t, s) w(\sigma(s)) \Delta s \\
+\int_{t_{0}}^{t} H(t, s) G(s) \frac{w(s)}{\delta(s)} \Delta s \\
\quad-\int_{t_{0}}^{t} H(t, s) Q(s) \frac{w^{2}(s)}{\delta^{2}(s)} \Delta s \\
=H\left(t, t_{0}\right) w\left(t_{0}\right)
\end{gathered}
$$




$$
\begin{aligned}
& -\int_{t_{0}}^{t} h(t, s) \sqrt{H(t, s)} w(\sigma(s)) \Delta s \\
& +\int_{t_{0}}^{t} H(t, s) G(s) \frac{w(s)}{\delta(s)} \Delta s \\
& -\int_{t_{0}}^{t} H(t, s) Q(s) \frac{w^{2}(s)}{\delta^{2}(s)} \Delta s .
\end{aligned}
$$

Using $\left(H_{5}\right)$ and Lemma 3, we get

$$
\begin{gathered}
\frac{1}{x(\tau(t))} \geq \frac{1}{x(\tau(\sigma(t)))}, \\
r(t) g\left(x(t), x^{\Delta}(t)\right) \geq\left(r(t) g\left(x(t), x^{\Delta}(t)\right)\right)^{\sigma} .
\end{gathered}
$$

Therefore,

$$
\begin{array}{rl}
\int_{t_{0}}^{t} L & H(t, s) q(s) \delta^{\sigma}(s) \alpha(\tau(s)) \Delta s \\
\leq & H\left(t, t_{0}\right) w\left(t_{0}\right) \\
& +\int_{t_{0}}^{t} \frac{G(s) H(t, s)+\delta(s) h_{-}(t, s) \sqrt{H(t, s)}}{\delta(s)} w(s) \Delta s \\
& -\int_{t_{0}}^{t} H(t, s) Q(s) \frac{w^{2}(s)}{\delta^{2}(s)} \Delta s \\
= & H\left(t, t_{0}\right) w\left(t_{0}\right) \\
& +\int_{t_{0}}^{t} \frac{G(s) \sqrt{H(t, s)}+\delta(s) h_{-}(t, s)}{\delta(s)} \sqrt{H(t, s)} w(s) \Delta s \\
& -\int_{t_{0}}^{t} H(t, s) Q(s) \frac{w^{2}(s)}{\delta^{2}(s)} \Delta s \\
= & H\left(t, t_{0}\right) w\left(t_{0}\right) \\
& +\int_{t_{0}}^{t} \frac{1}{4 Q(s)}+\int_{t_{0}}^{t}\left[\frac{\sqrt{H(t, s)}}{\delta(s)} \sqrt{Q(s)} w(s)-\frac{1}{2 \sqrt{Q(s)}}\right. \\
& \times[G(s) \sqrt{H(t, s)}-\delta(s) h(t, s)]^{2} \Delta s \\
& \times(t, s))]^{2} \Delta s \\
& \\
& \\
&
\end{array}
$$

$$
\begin{aligned}
& +\int_{t_{0}}^{t} \frac{K_{2} r(\tau(s))}{4 \delta^{\sigma}(s) \alpha(\tau(s)) \tau^{\Delta}(s)} \\
& \quad \times\left[G(s) \sqrt{H(t, s)}+\delta(s) h_{-}(t, s)\right]^{2} \Delta s
\end{aligned}
$$

Thus

$$
\begin{aligned}
\frac{1}{H\left(t, t_{0}\right)} \int_{t_{0}}^{t}\left[H(t, s) q(s) \delta^{\sigma}(s) \alpha(\tau(s))\right. \\
-\frac{\eta r(\tau(s))}{4 \delta^{\sigma}(s) \alpha(\tau(s)) \tau^{\Delta}(s)} \\
\left.\quad \times\left[G(s) \sqrt{H(t, s)}+\delta(s) h_{-}(t, s)\right]^{2}\right] \Delta s \\
<\frac{1}{L} w\left(t_{0}\right)<\infty,
\end{aligned}
$$

which is a contradiction to (58). This completes the proof.

Theorem 8. Assume that $\left(H_{1}\right)-\left(H_{5}\right)$ and (3) hold. If there exists a positive $\Delta$-differentiable function $\delta(t)$ such that (36) holds and

$$
\int_{t_{0}}^{\infty}\left[\frac{1}{r(t)} \int_{t_{0}}^{t} q(s) e_{-p / r}(t, \sigma(s)) \Delta s\right] \Delta t=\infty,
$$

then every solution of (1) is either oscillatory or converges to zero on $\left[t_{0}, \infty\right)_{\mathbb{T}}$.

Proof. Assume that (1) has a nonoscillatory solution $x(t)$ such that $x(t)>0, x(\tau(t))>0$ for all $t \in\left[t_{1}, \infty\right)_{\mathbb{T}}, t_{1} \in\left[t_{0}, \infty\right)_{\mathbb{T}}$. As in the proof of Lemma 3, we see that there exist two possible cases for the sign of $x^{\Delta}(t)$. When $x^{\Delta}(t)$ is eventually positive, the proof is similar to the proof of Theorem 4 . Next, suppose that $x^{\Delta}(t)<0$ for $t \in\left[t_{1}, \infty\right)_{\mathbb{T}}$. Then, $x(t)$ is decreasing and $\lim _{t \rightarrow \infty} x(t)=b \geq 0$. Thus,

$$
x(\tau(t))>x(t)>x(\sigma(t)) \geq b \geq 0, \quad \forall t \in\left[t_{1}, \infty\right)_{\mathbb{T}} .
$$

Hence, by $\left(\mathrm{H}_{3}\right)$, we get

$$
f(x(\tau(t))) \geq L x(\tau(t)) \geq L b, \quad \forall t \in\left[t_{1}, \infty\right)_{\mathbb{T}} .
$$

Defining the function $u(t)=r(t) g\left(x(t), x^{\Delta}(t)\right)$, using (1) and (66), we get

$$
\begin{aligned}
u^{\Delta}(t) & =-\frac{p(t)}{r(t)} u(t)-q(t) f(x(\tau(t))) \\
& \leq-\frac{p(t)}{r(t)} u(t)-\operatorname{Lbq}(t), \quad \text { for } t \in\left[t_{1}, \infty\right)_{\mathbb{T}} .
\end{aligned}
$$

The inequality (67) is the assumed inequality of [18, Theorem 6.1]. All other assumptions of [18, Theorem 6.1]; for example, $-p / r \in \mathscr{R}$, are satisfied. Hence, the conclusion of [18, Theorem 6.1] holds; that is,

$$
\begin{aligned}
u(t) & \leq u\left(t_{1}\right) e_{-p / r}\left(t, t_{1}\right)-L b \int_{t_{1}}^{t} q(s) e_{-p / r}(t, \sigma(s)) \Delta s \\
& <-L b \int_{t_{1}}^{t} q(s) e_{-p / r}(t, \sigma(s)) \Delta s,
\end{aligned}
$$


for all $t \in\left[t_{1}, \infty\right)_{\mathbb{T}}$, and thus, for all $\xi \in\left[t_{1}, \infty\right)_{\mathbb{T}}$,

$$
\begin{aligned}
& \int_{t_{1}}^{\xi} x^{\Delta}(t) \Delta t \\
&<-\frac{L b}{K_{1}} \int_{t_{1}}^{\xi}\left(\frac{1}{r(t)} \int_{t_{1}}^{t} q(s) e_{-p / r}(t, \sigma(s)) \Delta s\right) \Delta t, \\
& K_{1}>0 .
\end{aligned}
$$

Assuming $b>0$ and using (64) in (69) yield $\lim _{r \rightarrow \infty} x(r)=$ $-\infty$, which is a contradiction to the fact that $x(t)>0$ for $t \in\left[t_{1}, \infty\right)_{\mathbb{T}}$. Thus, $b=0$ and then $\lim _{t \rightarrow \infty} x(t)=0$. This completes the proof.

Remark 9. Our results in this paper not only extend and improve some known results and show that some results of $[2,8-10,12,14]$ are special examples of our results but also unify the study of oscillation of second-order nonlinear delay differential equation with damping and second-order nonlinear delay difference equation with damping.

\section{Examples}

Example 1. Consider the second-order delay 2-difference equation with damping

$$
\begin{aligned}
& \left(t^{2 / 3} \frac{x^{2}(t)\left(x^{\Delta}(t)\right)^{3}}{2+x^{2}(t)\left(x^{\Delta}(t)\right)^{2}}\right)^{\Delta}+\frac{1}{t^{2}}\left(\frac{x^{2}(t)\left(x^{\Delta}(t)\right)^{3}}{2+x^{2}(t)\left(x^{\Delta}(t)\right)^{2}}\right) \\
& +\frac{1}{t^{4 / 3}} x\left(\frac{t}{2}\right)=0, \quad t \in \overline{2^{\mathbb{Z}}}, t \geq t_{0}:=2 .
\end{aligned}
$$

Here,

$$
\begin{gathered}
r(t)=t^{2 / 3}, \quad p(t)=\frac{1}{t^{2}}, \quad q(t)=\frac{1}{t^{4 / 3}}, \\
g\left(x, x^{\Delta}\right)=\frac{x^{2}(t)\left(x^{\Delta}(t)\right)^{3}}{2+x^{2}(t)\left(x^{\Delta}(t)\right)^{2}},
\end{gathered}
$$

The conditions $\left(H_{1}\right),\left(H_{5}\right)$ are clearly satisfied, $\left(H_{3}\right)$ and $\left(H_{4}\right)$ hold with $L=K_{2}=1, K_{1}=1 / 2$, and $\left(H_{2}\right)$ is satisfied as

$$
1-\mu(t) \frac{p(t)}{r(t)}=1-t \frac{1}{t^{8 / 3}}=1-\frac{1}{t^{5 / 3}}>0, \quad \text { for } t \geq 2 \text {. }
$$

By Lemma 2, we get

$$
\begin{aligned}
e_{-p / r}(t, 2) & \geq 1-\int_{2}^{t} \frac{p(s)}{r(s)} \Delta s \\
& =1-\int_{2}^{t} s^{-8 / 3} \Delta s=1-\frac{t^{-5 / 3}-2^{-5 / 3}}{2^{-5 / 3}-1} \\
& =\frac{t^{-5 / 3}+1-2^{-2 / 3}}{1-2^{-5 / 3}} \geq t^{-5 / 3}+1-2^{-2 / 3} \\
& >1-2^{-2 / 3}>\frac{1}{3},
\end{aligned}
$$

for $t \geq 2$, so that

$$
\begin{aligned}
\int_{2}^{t} & {\left[\frac{1}{r(s)} e_{-p / r}(s, 2)\right] \Delta s } \\
& =\int_{2}^{t} s^{-2 / 3} e_{-p / r}(s, 2) \Delta s \geq \frac{1}{3} \int_{2}^{t} s^{-2 / 3} \Delta s \\
& =\frac{1}{3} \frac{t^{1 / 3}-2^{1 / 3}}{2^{1 / 3}-1} \longrightarrow \infty, \quad \text { as } t \longrightarrow \infty .
\end{aligned}
$$

Hence, (2) is satisfied. Then,

$$
\begin{aligned}
R(t) & =k r(t) \int_{2}^{t} \frac{\Delta s}{r(s)} \\
& =\frac{t^{2 / 3}}{2} \int_{2}^{t} \frac{\Delta s}{s^{2 / 3}} \geq \frac{1}{2}(t-2), \quad \text { for } k=\frac{1}{2}
\end{aligned}
$$

Therefore,

$$
\alpha(t)=\frac{R(t)}{R(t)+\mu(t)}=\frac{R(t)}{R(t)+t}>\frac{t-2}{3 t-2},
$$

and then we can find $0 \leq \lambda<1$ such that

$$
\alpha(t)>\frac{\lambda}{3}
$$

$$
\begin{aligned}
& \text { If } \delta(t)=1 \text {, then } \\
& \begin{aligned}
& \int_{2}^{t}[\alpha(\tau(s)) q(s) \\
&-\frac{\eta r(\tau(s))}{4 \alpha(\tau(s)) \tau^{\Delta}(s)} \\
&\left.\times\left(\frac{\delta^{\Delta}(s)}{\delta^{\sigma}(s)}-\frac{p(s)}{r(s)} \alpha(\tau(s))\right)^{2}\right] \delta^{\sigma}(s) \Delta s \\
&> \frac{\lambda}{3}\left[\int_{2}^{t} s^{-4 / 3} \Delta s-\frac{1}{2^{5 / 3}} \int_{2}^{t} s^{-14 / 3}\right] \Delta s \\
&= \frac{\lambda}{3}\left[\frac{t^{-1 / 3}-2^{-1 / 3}}{2^{-1 / 3}-1}-\frac{1}{2^{5 / 3}} \frac{t^{-11 / 3}-2^{-11 / 3}}{2^{-11 / 3}-1}\right] \longrightarrow \infty \\
&
\end{aligned}
\end{aligned}
$$

Hence, according to Theorem 4, every solution of (70) is oscillatory on $\left[t_{0}, \infty\right)_{\mathbb{T}}$.

Example 2. Consider the second-order nonlinear delay dynamic equation with damping

$$
\begin{aligned}
& \left(\frac{\sin ^{2}(x(t))}{1+x^{2}(t)} x^{\Delta}(t)\right)^{\Delta}+\frac{\beta}{t \sigma(t)}\left(\frac{\sin ^{2}(x(t))}{1+x^{2}(t)} x^{\Delta}(t)\right)^{\Delta} \\
& \quad+\frac{\lambda}{t \alpha(t)} x(t)\left(x^{2}(t)+1\right)=0, \quad \text { for } t \in\left[t_{0}, \infty\right)_{\mathbb{T}}, t_{0} \geq 0,
\end{aligned}
$$

where $\lambda>0$ and $0<\beta<t_{0} \leq t$. 
Here,

$$
\begin{gathered}
r(t)=1, \quad p(t)=\frac{\beta}{t \sigma(t)}, \quad q(t)=\frac{\lambda}{t \alpha(t)}, \\
g\left(x, x^{\Delta}\right)=\frac{\sin ^{2}(x(t))}{1+x^{2}(t)} x^{\Delta}(t), \quad f(x)=x\left(x^{2}+1\right), \\
\tau(t)=t .
\end{gathered}
$$

The conditions $\left(H_{1}\right),\left(H_{5}\right)$ are clearly satisfied, $\left(H_{3}\right)$ and $\left(H_{4}\right)$ hold with $L=K_{2}=1$, and $\left(H_{2}\right)$ is satisfied as

$$
1-\mu(t) \frac{p(t)}{r(t)}=1-\frac{\beta \mu(t)}{t \sigma(t)}>0, \quad \text { for } 0<\beta<t_{0} \leq t
$$

By Lemma 2, we get

$$
\begin{aligned}
\int_{t_{0}}^{\infty} & {\left[\frac{1}{r(t)} e_{-p / r}(t, 2)\right] \Delta t } \\
\geq & \int_{t_{0}}^{\infty}\left[1-\int_{t_{0}}^{t} \frac{p(s)}{r(s)} \Delta s\right] \Delta t \\
= & \int_{t_{0}}^{\infty}\left[1-\beta \int_{t_{0}}^{t}\left(\frac{-1}{s}\right)^{\Delta} \Delta s\right] \Delta t \\
= & \int_{t_{0}}^{\infty}\left[1-\beta\left(\frac{-1}{t}+\frac{1}{t_{0}}\right)\right] \Delta t \\
= & \int_{t_{0}}^{\infty}\left[\left(1-\frac{\beta}{t_{0}}\right)+\frac{\beta}{t}\right] \Delta t .
\end{aligned}
$$

Hence,

$$
\begin{aligned}
& \int_{t_{0}}^{\infty}\left[\frac{1}{r(t)} e_{-p / r}(t, 2)\right] \Delta t \\
& \quad \geq\left(1-\frac{\beta}{t_{0}}\right) \int_{t_{0}}^{\infty} \Delta t+\beta \int_{t_{0}}^{\infty} \frac{1}{t} \Delta t=\infty .
\end{aligned}
$$

Therefore, (2) is satisfied. Then,

$$
\begin{aligned}
\alpha(t) & =\frac{R(t)}{R(t)+\mu(t)}=\frac{R(t)}{R(t)+\sigma(t)-t} \\
& \leq \frac{R(t)}{R(t)+t-t} \leq 1, \quad \frac{1}{\sigma(t)} \leq \frac{1}{t}, \quad \text { for } \sigma(t) \geq t .
\end{aligned}
$$

If $H(t, s)=t$ and $\delta(t)=1$, then $h(t, s)=0, G(t)=$ $-\beta \alpha(\tau(t)) / t \sigma(t)$ and

$$
\begin{aligned}
& \limsup _{t \rightarrow \infty} \frac{1}{H\left(t, t_{0}\right)} \\
& \times \int_{t_{0}}^{t}[H(t, s) q(s) \alpha(\tau(s)) \\
& \quad-\frac{\eta r(\tau(s))}{4 \alpha(\tau(s)) \tau^{\Delta}(s)}
\end{aligned}
$$

$$
\begin{aligned}
& \left.\quad \times\left(\frac{G(s)}{\delta^{\sigma}(s)} \sqrt{H(t, s)}+\frac{\delta(s)}{\delta^{\sigma}(s)} h_{-}(t, s)\right)^{2}\right] \\
& \times \delta^{\sigma}(s) \Delta s \\
& =\limsup _{t \rightarrow \infty} \frac{1}{t} \int_{t_{0}}^{t} \frac{1}{s}\left(\lambda t-\frac{\beta^{2} \alpha(s)}{4 s \sigma^{2}(s)} t\right) \Delta s \\
& \geq \limsup _{t \rightarrow \infty} \frac{1}{t} \int_{t_{0}}^{t} \frac{1}{s}\left(\lambda t-\frac{\beta^{2}}{4 s^{3}} t\right) \Delta s \\
& =\limsup _{t \rightarrow \infty} \int_{t_{0}}^{t} \frac{1}{s}\left(\lambda-\frac{\beta^{2}}{4 s^{3}}\right) \Delta s=\infty, \quad \text { for } \lambda>0 .
\end{aligned}
$$

Hence, according to Theorem 7, every solution of (79) is oscillatory on $\left[t_{0}, \infty\right)_{\mathbb{T}}$.

Remark 10. The results of [10] cannot be applied to equation (70) for $r(t)=t^{2 / 3}$ and $g\left(x, x^{\Delta}\right)=x^{2}(t)\left(x^{\Delta}(t)\right)^{3} /(2+$ $\left.x^{2}(t)\left(x^{\Delta}(t)\right)^{2}\right)$. Also, the results of [16] cannot be applied to (79) for $g\left(x, x^{\Delta}\right)=\left(\sin ^{2}(x(t)) /\left(1+x^{2}(t)\right)\right) x^{\Delta}(t)$. But, according to our work, both (70) and (79) are oscillatory.

\section{Conflict of Interests}

The authors declare that there is no conflict of interests regarding the publication of this paper.

\section{References}

[1] R. P. Agarwal, M. Bohner, D. O'Regan, and A. Peterson, "Dynamic equations on time scales: a survey," Journal of Computational and Applied Mathematics, vol. 141, no. 1-2, pp. $1-26,2002$.

[2] R. P. Agarwal, M. Bohner, and S. H. Saker, "Oscillation of second order delay dynamic equations," Canadian Applied Mathematics Quarterly, vol. 13, pp. 1-18, 2005.

[3] R. P. Agarwal, D. O'Regan, and S. H. Saker, "Philos-type oscillation criteria for second order half-linear dynamic equations on time scales," Rocky Mountain Journal of Mathematics, vol. 37, no. 4, pp. 1085-1104, 2007.

[4] H. A. Agwa, A. M. M. Khodier, and H. A. Hassan, "Oscillation of second-order nonlinear delay dynamic equations on time scales," International Journal of Differential Equations, vol. 2011, Article ID 863801, 15 pages, 2011.

[5] M. Bohner, "Some oscillation criteria for first order delay dynamic equations," Far East Journal of Applied Mathematics, vol. 18, no. 3, pp. 289-304, 2005.

[6] M. Bohner and S. H. Saker, "Oscillation of second order nonlinear dynamic equations on time scales," Rocky Mountain Journal of Mathematics, vol. 34, no. 4, pp. 1239-1254, 2004.

[7] L. Erbe, A. Peterson, and S. H. Saker, "Oscillation criteria for second-order nonlinear dynamic equations on time scales," Journal of the London Mathematical Society, vol. 67, no. 3, pp. 701-714, 2003.

[8] L. Erbe, A. Peterson, and S. H. Saker, "Oscillation criteria for second-order nonlinear delay dynamic equations," Journal of Mathematical Analysis and Applications, vol. 333, no. 1, pp. 505522, 2007. 
[9] S. R. Grace, R. P. Agarwal, M. Bohner, and D. O’Regan, "Philos type criteria for second-order half-linear dynamic equations," Mathematical Inequalities and Applications, vol. 14, no. 1, pp. 211222, 2011.

[10] G. S. Guseinov and B. Kaymakçalan, "On a disconjugacy criterion for second order dynamic equations on time scales," Journal of Computational and Applied Mathematics, vol. 141, no. 1-2, pp. 187-196, 2002.

[11] T. S. Hassan, "Oscillation criteria for half-linear dynamic equations on time scales," Journal of Mathematical Analysis and Applications, vol. 345, no. 1, pp. 176-185, 2008.

[12] Y. Şahiner, "Oscillation of second-order delay differential equations on time scales," Nonlinear Analysis: Theory, Methods and Applications, vol. 63, no. 5-7, pp. e1073-e1080, 2005.

[13] S. H. Saker, "Oscillation criteria of second-order half-linear dynamic equations on time scales," Journal of Computational and Applied Mathematics, vol. 177, no. 2, pp. 375-387, 2005.

[14] S. Sun, Z. Han, and C. Zhang, "Oscillation of second-order delay dynamic equations on time scales," Journal of Applied Mathematics and Computing, vol. 30, no. 1-2, pp. 459-468, 2009.

[15] Q. Zhang, "Oscillation of second-order half-linear delay dynamic equations with damping on time scales," Journal of Computational and Applied Mathematics, vol. 235, no. 5, pp. 1180-1188, 2011.

[16] Q. Zhang and L. Gao, "Oscillation of second-order nonlinear delay dynamic equations with damping on time scales," Journal of Applied Mathematics and Computing, vol. 37, no. 1-2, pp. 145158, 2011.

[17] Q. Zhang, L. Gao, and L. Wang, "Oscillation of second-order nonlinear delay dynamic equations on time scales," Computers and Mathematics with Applications, vol. 61, no. 8, pp. 2342-2348, 2011.

[18] M. Bohner and A. Peterson, Dynamic Equations on Time Scales: An Introduction with Applications, Birkhäuser, Boston, Mass, USA, 2001.

[19] M. Bohner and A. Peterson, Advances in Dynamic Equations on Time Scales, Birkhäuser, Boston, Mass, USA, 2003. 


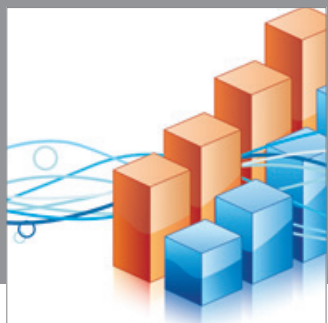

Advances in

Operations Research

mansans

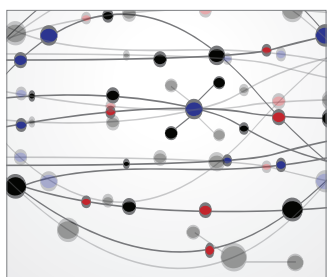

The Scientific World Journal
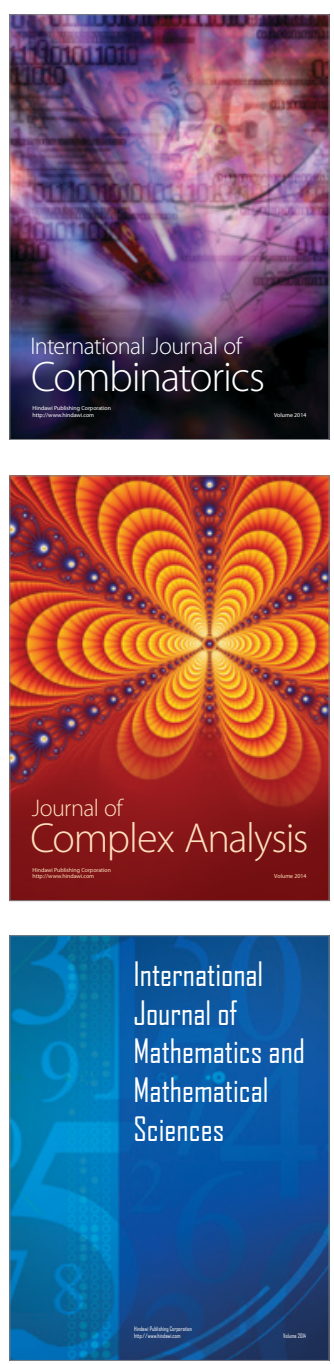
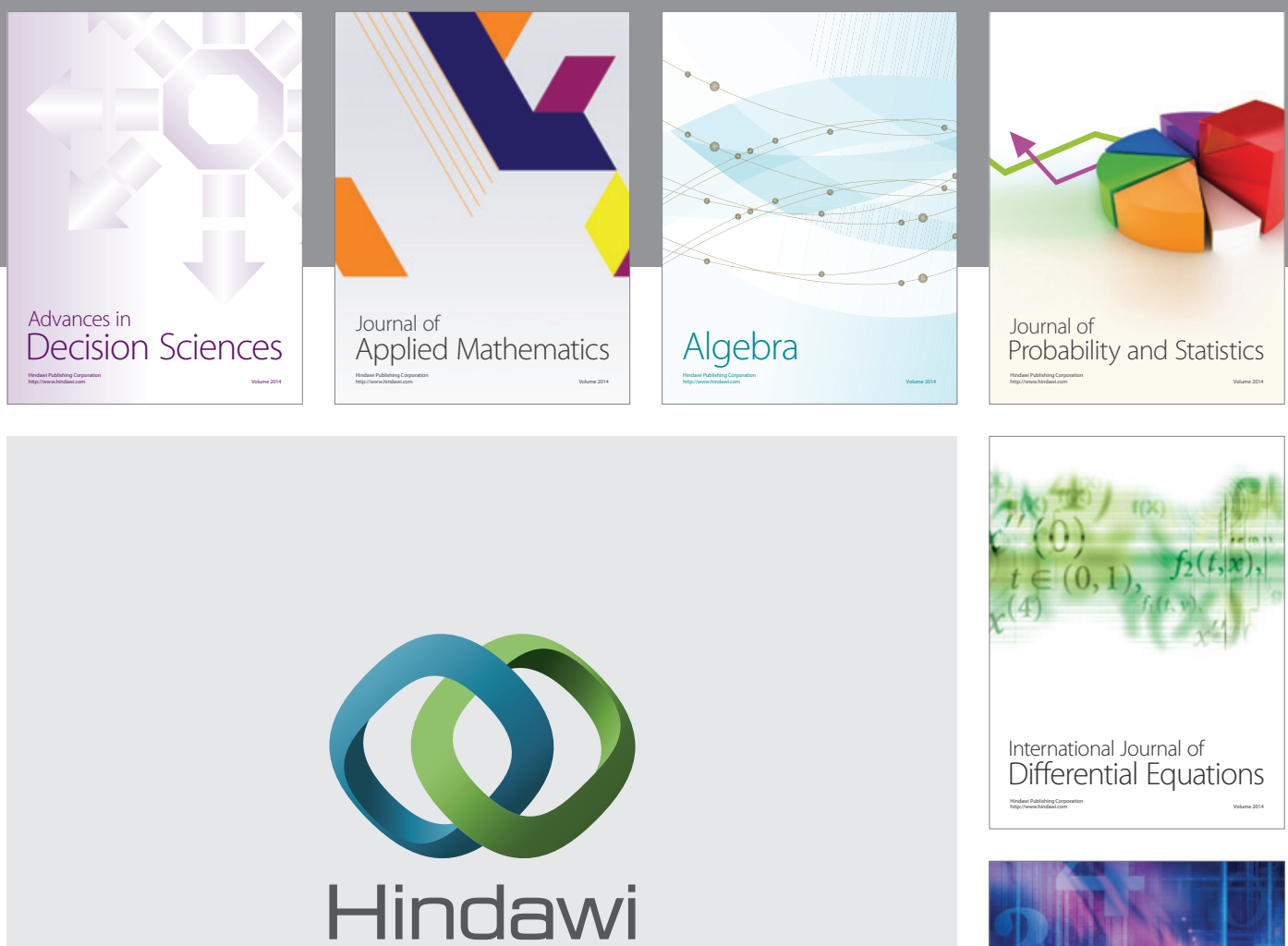

Submit your manuscripts at http://www.hindawi.com
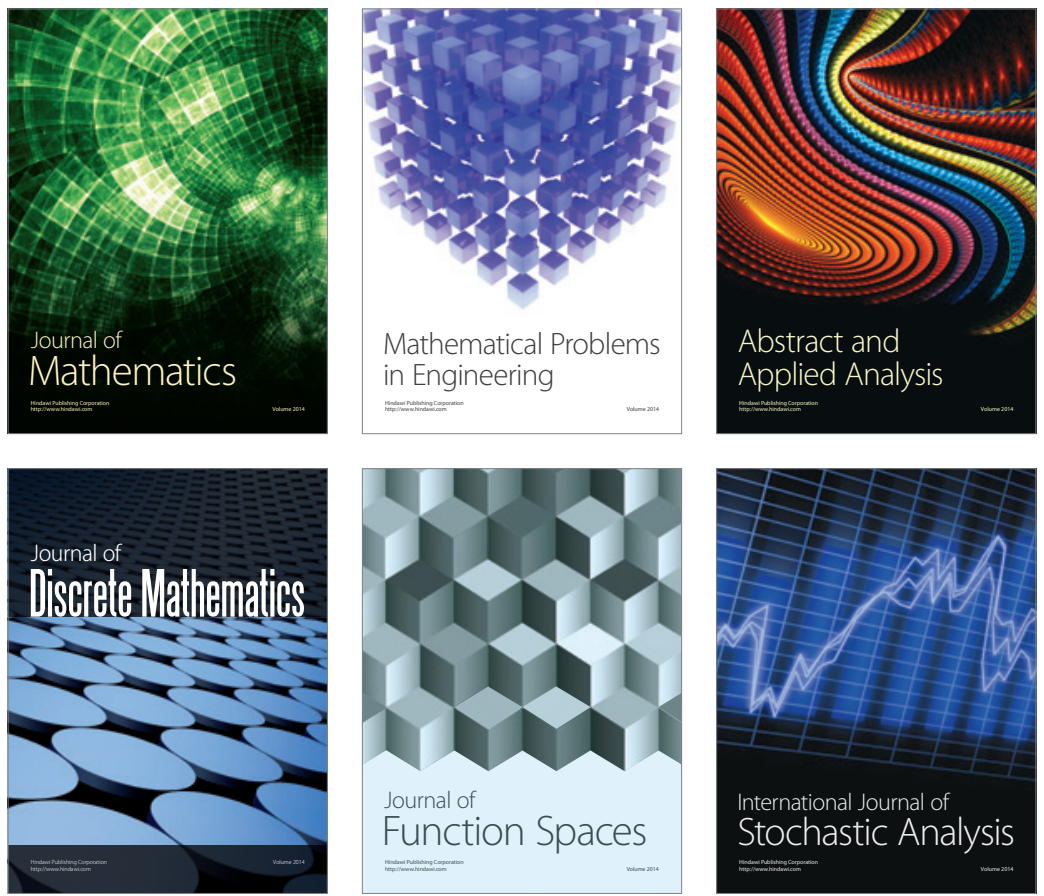

Journal of

Function Spaces

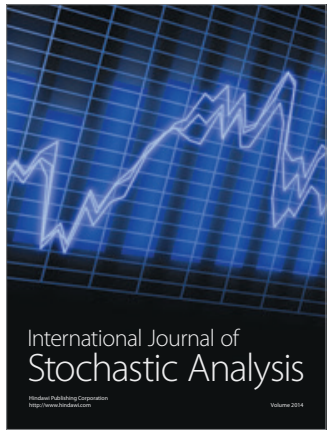

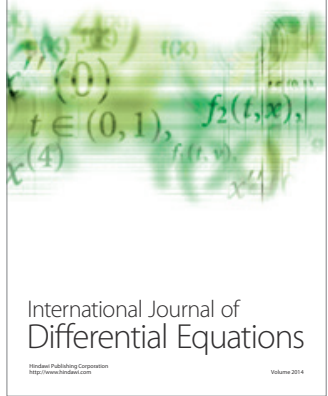
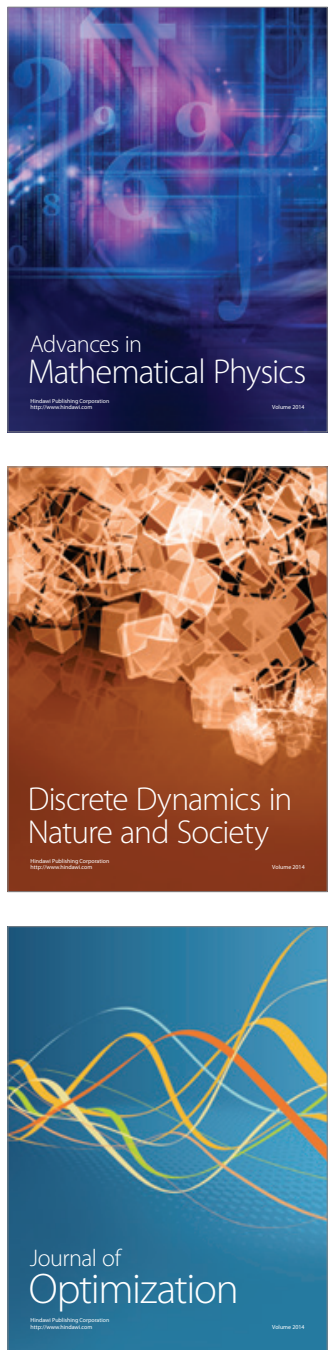